Págs.209-233

\title{
Entrevistas a equipos directivos
}

\section{Eva $M^{a}$ Tarín Hernández, Directora de CEIP Professor Ramiro Jover, 60 años, València}

Describe brevemente las características de la Asociación de Directoras y Directores de Infantil y Primaria del País Valenciano (ADIP-PV) que has presidido (objetivos, número de afiliados, iniciativas, etc). Indica cuánto tiempo llevas vinculada a esta asociación, así como en el centro educativo y desde cuándo eres directora del mismo.

ADIP-PV es un asociación de directoras y directores de la escuela pública, que nació en València hace más de una década y que luego se extendió a todo el País Valenciano. En la actualidad cuenta con más de 450 socios y socias. Entre sus objetivos se encuentran: ayudar y compartir informaciones, sugerencias, inquietudes y sobre todo coordinar todo esto en defensa y mejora de la escuela pública. Otro objetivo muy importante es transmitir a la Administración nuestras propuestas, nuestras inquietudes y nuestras necesidades, para que así, la gestión de los centros educativos mejore en cuanto a eficacia y eficiencia.

Llevo muchos años en equipos directivos y en mi última etapa, siendo directora desde hace 7 cursos en un colegio de València. He sido la presidenta de la Junta de València durante los últimos cuatro cursos, cargo que dejé al finalizar el curso anterior.

Explica brevemente cuáles son los cambios principales, a tu juicio, que ha generado la suspensión de la asistencia a clases presenciales, tanto para el profesorado como para el alumnado y las familias. ¿2Puedes describir brevemente tu rutina diaria en lo que se refiere a tu dedicación a la docencia no presencial, comentando también si ha aumentado o se ha alterado el tiempo de trabajo?

Desde el primer día de confinamiento, surgió una gran necesidad por parte del profesorado de poder llegar al alumnado a través de diferentes medios digitales. El profesorado que ya se manejaba más con recursos TIC, tenía el camino trazado, el que no, tuvo que buscar alternativas para poder llegar al alumnado. Las primeras semanas fueron frenéticas, se recibían un montón de recursos digitales, instrucciones de la administración que intentaban guiar la nueva situación, las horas de trabajo eran infinitas, no había horario. Desde Conselleria se nos ofrecían plataformas que colapsaban por el mayor número de usuarios al que se las estaba sometiendo.

Las direcciones nos vimos desbordadas con los vales que se emitieron para las becas de comedor, atendiendo las reclamaciones de las familias, gestionando las solicitudes para poder acceder a una de 
las plataformas que nos ofrecía Conselleria, web familia 2.0, coordinando las actuaciones pedagógicas, reuniones con uso de nuevas tecnologías... Las familias, escribían correos al centro, ya que no había posibilidad de conectar telefónicamente. Yo les contestaba unas veces vía correo electrónico otras tenía que llamar con teléfono personal y número oculto.

Las familias mostraban su necesidad de saber como podían resolver sus problemas administrativos, estaban tranquilas y a la vez impacientes por obtener soluciones. Siempre con buenas palabras y agradeciendo el trabajo que estábamos realizando.

El profesorado fue el que se encargó de contactar con las familias vía correo electrónico y WhatsApp mayoritariamente, ya que la plataforma web familia no era un recurso muy utilizado en nuestro centro.

Quizás sea un poco prematuro hacer una valoración al respecto, pero diversas consideraciones podríamos destacar: en primer lugar, la necesidad de utilizar con mayor eficiencia y eficacia las nuevas tecnologías, es decir, nos faltaba preparación y formación; en segundo lugar, se ha podido comprobar como gracias a las aplicaciones y recursos TIC (muchos de ellos de carácter privado, no autorizados por Conselleria) se ha podido dar la atención educativa necesaria y atender a las familias, dando una respuesta más dinámica e interactiva a las demandas reales de esta situación de alarma.

¿Cómo se ha llevado a cabo el proceso de adaptación a esta nueva situación, por parte de la comunidad educativa (profesorado, alumnado, familias)? ¿ ¿Destacarías alguna cuestión significativa en este proceso?

Sin duda, como ante cualquier otra situación nueva, hemos experimentado un poco de ansiedad (salvando las distancias) por la necesidad de dar una pronta respuesta a las familias, algunas de ellas en situaciones de riesgo o peligro de exclusión social, a través de unos medios tecnológicos con los cuales no estábamos suficientemente familiarizados. Pero tras las dos primeras semanas frenéticas, poco a poco hemos podido «normalizar» la situación.

Me gustaría destacar cómo se ha demostrado la unión de la comunidad educativa, a pesar de la distancia, a través de vídeos de agradecimiento y llamamiento a dar ánimos en todas direcciones, del Claustro a niños, niñas y familias, de las familias al profesorado, interacciones de la comunidad educativa en general dando ánimos para superar el confinamiento.

¿Cómo realizas en estas nuevas circunstancias las tareas de dirección del centro en el día a día, cómo te coordinas con el resto de directores y directores en la ADIP-PV? Las tareas de dirección siguen el curso normal y de forma satisfactoria gracias a los medios tecnológicos y las plataformas de gestión que utilizamos. Los equipos directivos solemos estar bastante bien comunicados e informados entre nosotros gracias también a los medios tecnológicos. Los grupos de WhatsApp, telegram son los más dinámicos. Como novedad hemos hecho las reuniones a través de vídeo conferencias con el Claustro, equipo directivo, equipo pedagógico, ciclos e inspección.

Hemos redireccionado el teléfono del colegio al personal y utilizamos la llamada oculta desde nuestro teléfono personal cuando es necesaria la comunicación telefónica.

Respecto a ADIP-PV durante esta nueva situación, a mi parecer, ha tenido dos momentos cruciales. En el primero, dejó a un lado los objetivos de la asociación para ponerse al lado de la administración. Tal vez el miedo a lo desconocido, fomentó esta unión de las juntas directivas con la administración. Este fue un momento tenso que generó crispación por las formas adoptadas y una fractura de la junta de València. 
El segundo momento ha sido la vuelta a la normalidad, generando vías más rápidas de participación y transparencia en las medidas a adoptar por ADIP-PV.

Nuestra vía de comunicación como asociación son grupos de WhatsApp de las juntas, moodle d'adippv socios y socias, y a partir de esta crisis, se ha formado un grupo de telegram para socios y socias de la provincia de València.

¿Qué valoración puedes realizar sobre la situación actual, y los cambios que ha generado, desde tu experiencia tanto en ADIP-PV, como en la dirección del centro?

Sin duda hemos visto la necesidad de articular unos mecanismos efectivos que transmitan el sentir y las propuestas de los socios y socias, con unos criterios claros de recogida de información que demuestren de forma clara y transparente el sentir mayoritario de la asociación.

Con respecto a los centros educativos, el paraguas jurídico es suficiente para atender esta nueva situación, aunque sin duda siempre hay aspectos a mejorar.

¿Qué dificultades destacarías a la hora de llevar a cabo tu cometido en la dirección del centro? ¿Qué dificultades habéis observado desde vuestra experiencia y conocimiento como miembros de ADIP-PV?

He echado en falta un teléfono móvil corporativo, hubiese sido el modo más rápido para aquellas familias que han necesitado contactar con el colegio, por los problemas comentados anteriormente sobre las incidencias de los vales del comedor. Estas familias son las que tienen menos capacidad de medios tecnológicos para comunicarse, muchas de ellas no tienen ni correo electrónico. Actualmente la Conselleria no autoriza la contratación de una línea móvil.

¿Señalarías algún tipo de cambio significativo en la relación profesional con el profesorado de tu centro?

El compromiso y la profesionalidad de los y las docentes, una vez más ha sido ejemplar.

El grupo de WhatsApp del claustro ha experimentado una actividad inusual, se han compartido tanto aspectos profesionales y humanos con una alta participación.

Ahora te pedimos que hagas un breve balance sobre cuestiones relativas a las respuestas ante una experiencia de confinamiento y de teletrabajo y los efectos de esta situación.

¿Encuentras diferencias en la respuesta del profesorado ante la situación actual? ¿̉Responde todo el profesorado de la misma manera a la hora de realizar la actividad docente en línea? Si la respuesta es diferente, ¿̇a qué crees que se debe? ¿Qué efectos produce esta situación en el profesorado?

La gran mayoría se ha volcado igual o más que antes y he notado que cada uno ha utilizado su capacidad TIC para contactar con el alumnado.

Ha habido una gran preocupación por todas aquellas familias más apartadas del grupo clase y se las ha integrado en el grupo de WhatsApp de la clase. 
¿Se ha puesto en marcha algún proceso de formación del profesorado para orientar su actividad docente ante el cambio producido por el paso de la docencia presencial a la docencia en línea? Si es así, ¿̇puedes hacer alguna valoración sobre esta experiencia?

Ha habido intercambios de información en cuanto a conocimiento de diferentes medios para dar respuesta a la necesidad creada por la nueva situación, Aules y Classroom entre otros.

El aislamiento ha generado más ganas de compartir, de alguna manera es querer saber del otro y llegar lo más lejos posible y al máximo de personas.

Compartimos muchísima información, tanto de recursos como de medios.

¿Qué respuestas estás encontrando por parte de las familias para acompañar las tareas educativas (deberes) de sus hijos? ¿̇Es la misma por parte de las familias? ¿Encuentras diferencias entre unas y otras? Si es así, ¿̇a qué crees que es debido?

La mayoría de las familias han tratado de contribuir a la normalidad coordinándose y contactando con los y las docentes, hay que entender que para todos ha sido una situación muy compleja y alarmante. De un día a otro cambia la manera de enseñar, el profesorado ya no está presente, están sus recomendaciones, la familia pasa a ser la que toma las riendas del proceso.

Hay familias que por sus condiciones socioeconómicas y culturales, no pueden liderar el proceso, no tienen capacidad ni medios. Hemos intentado llegar a ellas pero es muy complicado que sigan una rutina, anteriormente a esta crisis tampoco lo hacían.

¿Qué respuestas estás encontrando por parte del alumnado a la hora de realizar las tareas educativas cotidianas en una situación de educación en línea? ¿encuentras respuestas diferentes? Si es así, ¿a qué crees que es debido? ¿Qué consecuencias destacarías desde tu conocimiento y experiencia como directora del centro y como asociada de ADIP-PV?

Las respuestas del alumnado mayoritariamente han sido buenas, pero hay que recordar que no estábamos suficientemente preparados para abordar toda la enseñanza a través de medios tecnológicos, ni todas las familias, como he dicho anteriormente, tienen suficientes medios para dar una respuesta a los nuevos planteamientos. Esta situación nos ha hecho ver y padecer de nuestras carencias en cuanto a medios tecnológicos.

También habría que hacer un apartado especial para el alumnado con necesidades de apoyo educativo. Con estas criaturas es más difícil trabajar en la distancia, para ellos es fundamental tener un referente que les motive y acompañe en el proceso de aprendizaje.

¿Qué respuesta han recibido y están recibiendo los centros por parte de la administración educativa (consejería de educación, inspección educativa...) ante esta situación? La misma que antes de esta situación de alarma, pero con más periodicidad. Diariamente hemos recibido instrucciones que nos han guiado ante la nueva toma de decisiones. Los canales y la coordinación siguen siendo los mismos que antes e igual de válidos. 


\section{¿Cuáles son las principales conclusiones que extraes de esta experiencia educativa?}

Lo que hoy es válido, mañana no sirve, hemos de estar en constante renovación e innovación sin dejar de lado las emociones.

Somos afortunados de haber podido dar una respuesta en tan corto tiempo y debemos seguir esconzándonos y tratar de mejorar cada día.

Un nuevo día, un nuevo reto, hemos de estar en constante aprendizaje, aun sabiendo que los nuevos métodos no pueden suplir la parte emocional que conlleva la convivencia en un centro educativo.

Señala si crees que está teniendo algún impacto en términos de igualdad o desigualdad para el alumnado y, en caso afirmativo, en qué sentido.

Sin duda, las familias con menos recursos económicos y tecnológicos, en esta situación se han visto más perjudicadas, ya que no tienen la capacidad de respuesta que en esta situación se requiere.

He de reconocer y felicitar a la Conselleria de Educación, porque desde el primer momento, ha tratado de compensar esas desigualdades sociales y económicas en el alumnado más desfavorecido. Existe otro tipo de desigualdad, la cultural, al que es más difícil de proporcionar soluciones.

Ahora te pedimos que hagas un breve balance de esta experiencia de confinamiento y de teletrabajo educativo en relación a las siguientes cuestiones:

Principales debilidades y dificultades, situaciones de estrés, de conflicto entre el profesorado y entre el alumnado.

Tener los medios y los criterios organizativos suficientemente claros y delimitados para que, en cualquier caso, el proceso de enseñanza aprendizaje pueda continuar y ser atendido como corresponde para que llegue a la totalidad de nuestro alumnado.

Aspectos positivos, algo que puedas extraer como una ventaja en medio de esta situación compleja, alguna reflexión en términos de experiencia profesional y crecimiento personal.

La voluntad y empeño de ayudarnos entre todos y todas.

La reacción tan rápida en dar soluciones al problema de la distancia.

El compartir contenidos que antes eran más personalizados.

La mayor coordinación a la hora de emitir mensajes.

\section{Y en términos emocionales, afectivos y personales, ¿̇cómo lo estás viviendo? ¿̇cómo lo estás gestionando?}

Yo me considero una persona bastante estable emocionalmente, y eso me ha dado una tranquilidad que ha hecho que mi problema no fuese el COVID-19, sino dar solución a los nuevos problemas que se habían generado al suspender las clases.

Por esta misma razón estuve durante la primera semana, vacaciones de fallas, con la resolución de dos nuevas situaciones, una asistir a las familias en sus demandas e intentar convencer a mis compañeros de ADIP-PV, la necesidad de hacer una encuesta para demostrar a la administración que su decisión de asistir a los colegios directoras y directores, aún sin saber a qué, no era la adecuada. La primera cuestión fue generándome emociones positivas y la segunda, desgraciadamente me partió el alma, me encontré con personas que, a pesar de ser conocidas, para mí eran nuevas. 
Afortunadamente nuestras dificultades y complicaciones son asumibles y el tiempo hará que todo quede en su lugar aunque para ello haya que cambiar de sitio.

\section{Qué aprendizaje extraes de esta experiencia de cara al futuro.}

La capacidad de adaptación y el compromiso que todo tiene solución, y que por tanto, no podemos cruzarnos de brazos. Hemos de seguir buscando nuevas propuestas para adaptar nuestra escuela a las necesidades del siglo xxI, donde acompañemos a nuestro alumnado a sentir, a ser y a estar.

Te pedimos también que valores las consecuencias y el impacto educativo y social de esta crisis a corto y medio plazo, una vez que se supere la situación.

A nivel educativo, a corto plazo, debemos llevar un ritmo donde nadie quede por el camino, hacer propuestas que sean asumibles por la gran mayoría. Porque probablemente, si no actuamos en consecuencia y el acompañamiento no es el adecuado, la brecha que se abra entre nuestro alumnado será cada vez más grande y más difícil de eliminar. Hemos de pensar y hacer escuelas inclusivas, y solicitar a la administración los medios necesarios para ello, además individualmente, hemos de cambiar la mirada, las metodologías necesitan renovarse a través de consenso e instrucciones.

Socialmente deben hacerse actuaciones por parte de nuestros gobernantes, que ayuden a superar las necesidades socio económicas y culturales de una gran parte de la sociedad.

Finalmente, nos gustaría preguntarte por alguna otra cuestión, comentario, sugerencia, que quieras hacer para terminar

Me gustaría comentar la relación que hay entre el quedarse en casa para no contaminarnos de la COVID-19 y la suerte que ha tenido el planeta Tierra para mejorar la descontaminación. Cualquier crisis trae cosas positivas.

También creo que a nivel individual, las personas vamos a apreciar más las pequeñas cosas, salir a dar un paseo una tarde soleada, ver llover tras los cristales, bajar al parque con nuestros hijos e hijas... 


\section{Director del IES La Laboral de La Laguna, 46 años, Comunidad Autónoma Canaria}

Describe brevemente las características del centro educativo que diriges (nombre, ubicación, niveles educativos, número de profesorado y de alumnado, perfil aproximado del profesorado -sexo, edad-y del estudiantado -variables socioeconómicas, población inmigrante, etc.-. Indica cuánto tiempo llevas trabajando en el centro educativo y desde cuándo eres director del mismo.

Nombre del centro: IES La Laboral de La Laguna (Código de centro: 38002791).

Ubicación: Avda. Lora y Tamayo 2, San Cristóbal de La Laguna, Santa Cruz de Tenerife, Comunidad Autónoma Canaria.

Enseñanzas: 66 grupos repartidos en ESO, Bachillerato (Todos los itinerarios), Formación Profesional Básica (Electricidad y Servicios Administrativos), Formación Profesional de Grado Medio y Superior en presencial y en semipresencial (Familias profesionales de Administración y Empresas, Comercio y Marketing, Electricidad y Electrónica y Servicios Socioculturales y a la Comunidad) y Enseñanzas Deportivas.

Docentes: 128 con perfil aproximado (51\%mujeres) de 50\% con plaza definitiva, $25 \%$ profesorado funcionario con comisiones y resto funcionarios interinos. La edad media es cercana a los 50 años.

Alumnado: 1884 . De ellos cerca de 740 estudian ESO y Bachillerato y unos 1150 Formación Profesional (550 en régimen semipresencial en horario de noche). Las edades son dispares, ya que incorporamos a alumnado desde los doce años hasta la edad adulta. En el turno de mañana coincide alumnado de ESO, Bachillerato y ciclos formativos de grado superior y medio y en la tarde grado superior con FPB. El nivel socioeconómico de las familias es de nivel medio, con un 10\% de alumnado con dificultades económicas. El alumnado extranjero es el 5\% (102) con 31 nacionalidades (Venezolanos e italianos a la cabeza).

Director: trabajando en el centro 6 años, 1 de ellos como vicedirector y los otros 5 como director.

Explica brevemente cuáles son los cambios principales que, a tu juicio, ha generado la suspensión de la asistencia a clases presenciales, tanto para el profesorado como para el alumnado.

Los principales cambios han sido de carácter relacional y de rutinas de trabajo en ambos grupos (docentes y alumnado). Primero por los cambios en la metodología de trabajo, y segundo por la conciliación familiar para poder preparar y desarrollar esa metodología que se hace necesaria.

¿Cómo se ha llevado a cabo el proceso de adaptación a esta nueva situación, por parte de la comunidad educativa (profesorado, alumnado, familias)? ¿ ¿Destacarías alguna cuestión significativa en este proceso?

Utilizando los medios de comunicación oficiales del centro, siendo estos el correo corporativo (gmail con dominio propio para toda la comunidad educativa) y la APP MiColegioApp para las familias de la ESO, FPB y Bachillerato. Con estas herramientas y la formación del profesorado en los últimos 3 años, donde se les ha formado en metodologías de trabajo online, se ha conseguido conectar sin problemas con el alumnado. Lo único a destacar ha sido volcarnos en dos líneas de trabajo, la primera con los docentes que no tenían la formación y recursos para poder llevar a cabo esta enseñanza no presencial, y la segun- 
da, llegar al alumnado sin recursos (10\% del total del alumnado) a través de un seguimiento estricto por parte de los tutores y tutoras, todo ello para evitar las diferencias que ocasiona la brecha digital. También hemos querido adaptarnos con la información que se le traslada a toda la comunidad educativa, pasando de una hoja informativa semanal (SemanaBoral), a un boletín diario en periodo de confinamiento (\#NoticieroBoralDesdeCasa) (http://www.lalaboral.org/actividades/hoja-semanal/). En esa transformación se han introducido aspectos destacados en periodo de confinamiento, relativos al funcionamiento del centro, continuidad pedagógica, propuestas a la comunidad educativa (lectura, hábitos saludables, vídeos del centro...) e información actualizada de interés a las familias y alumnado.

\section{¿Cómo realizas en estas nuevas circunstancias las tareas de dirección del centro en} el día a día?

Nuestros recursos facilitan esta tarea. La herramienta GSuite incorporada a nuestro correo corporativo de Gmail, permite el uso de Meet, como aplicación de videoconferencia que nos permite estar en contacto constante a través de reuniones telemáticas en las que se han realizado profundas reflexiones constantes para tomar las decisiones que han terminado en instrucciones constantes al profesorado. Únicamente al principio del confinamiento se tuvo que ir al centro para trabajar con las aplicaciones in situ al no tener conexión remota, pero en una semana se pudo contar con el VPN que nos permitió al equipo directivo trabajar en remoto con estas y así gestionar todo sin problemas.

¿Qué valoración puedes realizar sobre la situación actual, y los cambios que ha generado, desde tu experiencia en la gestión y dirección del centro?

Inicialmente nos creó muchas dudas a la hora de tomar decisiones por la incertidumbre de las instrucciones dadas por parte de los órganos competentes (Consejería de Educación, Universidades, Cultura y Deportes del Gobierno de Canarias). Aún así, las decisiones tomadas, tras profundas reflexiones, creemos que han sido las acertadas. Esto nos refuerza como equipo, ratificando que los procedimientos y el trabajo en equipo que se está realizando es efectivo desde el punto de vista de la gestión. Estos cambios obligatorios, generados por el confinamiento, nos confirman que el profesorado debe estar formado en estas metodologías y que se debe hacer un esfuerzo en hacer desaparecer la brecha tecnológica en las familias más desfavorecidas para compensar la desigualdad y fomentar el apoyo a la diversidad. Tenemos la tarea hecha desde el primer aspecto (formación del profesorado) pero falta llegar a todo al alumnado (aunque nuestro centro es baja la afectación).

\section{¿Qué dificultades destacarías a la hora de llevar a cabo tu cometido en la dirección del centro?}

El gran volumen de alumnado y profesorado dificulta el poder llegar a todos, pero nuestros medios de comunicación han posibilitado este aspecto. Por otro lado, siempre hay docentes, a causa de esta gran heterogeneidad, que no están de acuerdo con las decisiones adoptadas y que entorpecen que la ejecución de estas indicaciones se realicen de manera fluida, aunque hay que reconocer que provocan una mayor reflexión y depuración a la hora de definir las instrucciones.

\section{¿Señalarías algún tipo de cambio significativo en la relación profesional con el profe- sorado de tu centro?}

Significativo no, pero se ha observado una respuesta espectacular por parte del profesorado, con apoyo 
e implicación total para sacar adelante esta situación tan compleja, a pesar de reticencias iniciales que terminaron por desaparecen, buscando una solución conjunta. Esto evidentemente está generando una mejor empatía y relación profesional entre todos los miembros del claustro.

Ahora te pedimos que hagas un breve balance sobre cuestiones relativas a las respuestas ante una experiencia de confinamiento y de teletrabajo y los efectos de esta situación.

¿Encuentras diferencias en la respuesta del profesorado ante la situación actual? ¿ Responde todo el profesorado de la misma manera a la hora de realizar la actividad docente en línea? Si la respuesta es diferente, ¿̇a qué crees que se debe? ¿Qué efectos produce esta situación en el profesorado?

Sí que hay diferencias, pero es muy bajo el porcentaje de docentes que no se adaptan a esta nueva situación. Unos pocos porque no creen que se pueda aplicar este tipo de enseñanza en estos momentos y no realizan adaptaciones metodológicas ante escasos recursos del alumnado (por ejemplo solicitar tareas que no se pueden realizar con móviles), y otros porque no tienen o recursos o formación para ello. Este escaso porcentaje de docentes, produce respuesta negativa a cómo se realiza el liderazgo por parte del equipo directivo, apoyándose en aspectos normativos que puede hacer que otros docentes con dudas puedan postularse en su posicionamiento. Pero repito, es muy poco el profesorado al que le ocurre esto y todos al final se han adaptado de manera rápida y eficiente.

Por otro lado, aunque una parte importante del profesorado y del alumnado conociéramos y tuviéramos implementadas las herramientas de teleformación, lo que ha sido fundamental para poder organizar una respuesta automática a esta situación, siempre se usaron para apoyar la enseñanza presencial (B-learning/Enseñanza Mixta) y el uso y el enfoque metodológico han cambiado para la enseñanza a distancia. Es importante que se produzca esta reflexión, que cada equipo educativo organice colegiadamente en qué tiempos van a llegar las tareas de cada materia, cuánto deben durar éstas, cómo plantearlas en aras a que una comunicación imperfecta no sea un hándicap para la realización por parte del alumnado.

\section{¿Se ha puesto en marcha algún proceso de formación del profesorado para orientar su actividad docente ante el cambio producido por el paso de la docencia presencial a la docencia en línea? Si es así, ¿̇puedes hacer alguna valoración sobre esta expe- riencia?}

Este centro, como se comentó, lleva 3 años con un Plan de Formación con varias líneas de trabajo, pero con énfasis en metodologías TIC usando aplicaciones como Classroom, Additio, GSuite, etc. Para los docentes que no se apuntaron en estos planes anuales y para los de nueva incorporación, se han proporcionado enlaces a autoformaciones y videotutoriales que facilitan el uso de estas herramientas comentadas que ya tienen disponibles. Para ello la figura del coordinador TIC del centro ha sido crucial, enviando información constante sobre estos temas y siendo quien responda a las dudas relacionadas con estos temas. La valoración desde este punto de vista ha sido muy positiva, reflejándose en la excelente respuesta del profesorado. 
¿Qué respuestas estás encontrando por parte de las familias para acompañar las tareas educativas de los hijos escolarizados en la educación obligatoria? ¿̇Es la misma por parte de las familias? ¿Encuentras diferencias entre unas y otras? Si es así, ¿a qué crees que es debido?

Evidentemente la respuesta va en función del nivel socioeconómico de cada familia. La familia media descrita está respondiendo bien, intentando ayudar a sus hijos en las tareas diarias en la medida de lo posible. Las familias de un nivel socioeconómico más elevado suelen apoyar más a sus hijos y contactar más con los tutores y docentes, siendo más exigentes y más críticos en cuento al exceso de tareas y la calidad ofrecida por los docentes en esta fase. Las familias de bajo nivel socioeconómico, al no tener recursos ni conocimiento para comunicarse por esta vía tecnológica es más complicado contactar con ellos, y/o muestran desinterés por ello. De todas formas, en su gran mayoría muestran interés para que sus hijos puedan realizar sus tareas a pesar de las dificultades de su situación. Otros aspectos que condicionan, como es lógico, son la edad del alumnado y los estudios que está realizando. No es lo mismo la respuesta en familias de alumnado de $1^{\circ}$ de las ESO que la de un alumno de formación profesional semipresencial que estudia en el nocturno con 43 años y padre de familia.

¿Qué respuestas estás encontrando por parte de las y los estudiantes a la hora de realizar las tareas educativas cotidianas en una situación de educación en línea? ¿encuentras respuestas diferentes? Si es así, ¿̇a qué crees que es debido? ¿Qué consecuencias destacarías desde tu conocimiento y experiencia como director del centro? La respuesta va en la misma línea de la pregunta anterior, donde, como era de esperar, el alumnado desmotivado y/o disruptivo en el aula también lo está demostrando desde sus casas. Con todo ello, la nota positiva es que alumnado que en clase se dispersaba por las relaciones con sus compañeros, y que estaban teniendo un rendimiento bajo, están teniendo una respuesta positiva, entre otras cosas por un aumento de la motivación hacia el trabajo, derivada de la ausencia de otros factores que desviaban su atención.

\section{¿Qué respuesta están recibiendo los centros por parte de la administración educativa (consejería de educación, inspección educativa...) ante esta situación?}

Esta pregunta es complicada de responder. La impresión de los centros ante esto es muy variada. Desde mi punto de vista están haciendo lo que pueden respondiendo lo antes posible a las demandas de cada comunidad educativa, pero la inestabilidad de esta situación y la complejidad de cómo afrontar el futuro está generando instrucciones muy interpretables y que no dejan avanzar en lo que muchos centros podrían hacer. Esto responde a la grandísima heterogeneidad de los centros educativos en función de las enseñanzas, ubicación del centro, perfil del alumnado, política de enseñanza, formación de los docentes, etc., donde en algunos centros es muy complicado poder llevar a cabo una enseñanza online y en otros es muy factible.

Por parte de la inspección educativa, con lo que respecta a la inspectora de nuestra zona, está en contacto con nosotros intentando responder a nuestras dudas mediante asesoramiento constante y prestando una ayuda esencial en nuestro avance como centro. 


\section{¿Cuáles son las principales conclusiones que extraes de esta experiencia educativa?}

Desde un centro como el nuestro, la conclusión principal es que estamos preparados para este tipo de situaciones y que debemos disponernos con mayor preparación y recursos para los que más lo necesitan para asegurar la equidad. Por otro lado, hay que salvar el reto de la evaluación. El gran freno en todo este proceso de confinamiento y educación no presencial ha sido «cómo evaluar a nuestro alumnado», hasta el punto de que los países del mismo entorno estén tomando decisiones totalmente diferentes, como es el caso de Italia, valorando el aprobado general, o el de España, pendiente de tomar decisiones tras escrito de asesoramiento del Consejo Escolar del Estado al Ministerio de Educación en donde se aconseja seguir con el proceso de evaluación del alumnado con condiciones que permitan asegurar la equidad y sin avanzar en contenidos.

Señala si crees que está teniendo algún impacto en términos de igualdad o desigualdad para el alumnado y, en caso afirmativo, en qué sentido.

En mi opinión sí que está generando un impacto en términos de igualdad (más bien equidad), al evidenciar que la brecha digital está presente y que se están tomando medidas y decisiones para compensarlas. Creo que sacaremos provecho de ello a modo de ayudas a las familias en cuanto a conectividad y recursos TIC.

Ahora te pedimos que hagas un breve balance de esta experiencia de confinamiento y de teletrabajo educativo en relación a las siguientes cuestiones:

Principales debilidades y dificultades, situaciones de estrés, de conflicto entre el profesorado y entre el alumnado.

Entre las principales debilidades, tuvimos la descoordinación inicial de los trabajos y tareas solicitadas al alumnado, corregida posteriormente con decisiones tomadas por los equipos educativos con la supervisión de los tutores, frenando la tensión de las familias (impacto inicial del confinamiento por la conciliación familiar y las tareas de los hijos). Por ello, la dificultad principal (aparte de la escasez de recursos disponibles en un $10 \%$ de las familias) es la conciliación familiar, tanto en casa de los docentes como la del alumnado. A veces el acceso a los recursos y las clases online se hacen difíciles realizando esto de manera asíncrona para salvar esta dificultad.

Una de las conclusiones que podemos sacar de este análisis, es que en esta situación se ha puesto en valor la necesidad de la acción tutorial como líder de cada equipo docente y como apoyo al alumnado y a las familias en las orientaciones para el enfoque del estudio. Ya vienen definidas en sus competencias, pero creo que es importante darle aún más valor al tutor/a.

Aspectos positivos, algo que puedas extraer como una ventaja en medio de esta situación compleja, alguna reflexión en términos de experiencia profesional y crecimiento personal.

Sigo pensando que se ha demostrado que se está preparado para este tipo de situaciones en muchos centros. Sin embargo, no creo que el inmovilismo sea la solución para solventarlas. Hay que tomar decisiones arriesgadas, pero con una gran reflexión por parte de los líderes pedagógicos que permitan avanzar y solucionar los problemas que surjan. Si se salvan estos inconvenientes, se podrá evaluar al alumnado, avanzando en contenidos no dados en enseñanza presencial, principal problema que se ha generado para un correcto cierre del curso académico. 


\section{Y en términos emocionales, afectivos y personales, ¿̇cómo lo estás viviendo? ¿̇cómo lo estás gestionando?}

Desde ese punto de vista no estoy teniendo ningún problema. El equipo directivo está trabajando como equipo (nos reunimos todos los días mediante Meet), y esto facilita estar fuerte al saber que hay un equipo detrás que respalda todas las decisiones. También ayuda ver que la práctica totalidad del profesorado está trabajando con una vocación increíble, lo que hace sentirte orgulloso del trabajo de tu equipo.

\section{Qué aprendizaje extraes de esta experiencia de cara al futuro.}

Esta situación extrema nos hace realizar un balance de lo ocurrido. Valorar en lo que se ha fallado y en lo que se ha hecho bien. Con ello buscar propuestas de mejora para que la próxima vez estemos preparados. Por ejemplo seguir invirtiendo en formación TIC, en recursos, buscar ayudas de conectividad a nuestro alumnado más necesitado, etc. Quedan otros problemas por buscar soluciones, como por ejemplo salvar la formación que exige presencialidad, como son los módulos eminentemente prácticos en familias profesionales como Electricidad, Servicios Socioculturales y a la Comunidad, o en Enseñanzas Deportivas, como es el caso de nuestro centro, y cómo no, la FCT (Formación en Centros de Trabajo).

\section{Te pedimos también que valores las consecuencias y el impacto educativo y social de} esta crisis a corto y medio plazo, una vez que se supere la situación

En mi opinión, esto acelerará el debate sobre el nuevo paradigma de la educación virtual interactiva, hacia una enseñanza más digital, no solo porque nos lo reclaman los avances y la realidad de esta sociedad digitalizada, sino en previsión de nuevas pandemias o situaciones que obliguen a confinamientos en un futuro. Para ello, creo que saldrán nuevas becas y ayudas destinadas a que todo el alumnado de la enseñanza obligatoria tenga acceso a datos y recursos tecnológicos (no sé cómo ni cuándo, pero será la tendencia), para hacer desaparecer la brecha digital y tener acceso a la enseñanza online. Evidentemente, no desaparecerá la enseñanza presencial, sería un grandísimo error limitar o no educar en valores, y no permitir la adquisición de competencias sociales y cívicas, más en una sociedad latina como la española, aunque tristemente tendremos que cambiar hábitos en nuestro país por este nuevo tipo de amenazas, que como hemos comprobado, no son de ciencia ficción.

Atentamente,

Fernando González Villavecchia Director del IES La Laboral de La Laguna 38002791@gobiernodecanarias.org director@ieslalaboraldelalaguna.es Twittery Facebook:@LaboralLaguna www.lalaboral.org

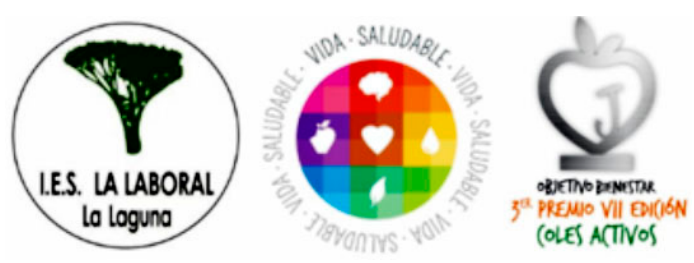




\section{Iñigo Beristain, Director del CEIP Público Labastida, 46 años, Labastida, Álava}

Describe brevemente las características del centro educativo que diriges (nombre, ubicación, niveles educativos, número de profesorado y de alumnado, perfil aproximado del profesorado -sexo, edad-y del estudiantado -variables socioeconómicas, población inmigrante, etc.-. Indica cuánto tiempo llevas trabajando en el centro educativo y desde cuándo eres director del mismo.

Nombre de centro: Colegio Público de Labastida ubicado en Labastida (Álava).

Enseñanzas: Educación Infantil (en Euskadi desde los 2 años), Educación Primaria.

Docentes: en total somos 17 profesionales entre maestros, maestras y educadoras, de los cuales 14 estamos con jornada completa. El perfil del profesorado: de 17, 13 son mujeres y 4 hombres. Con una edad media de 39 años aproximadamente.

Alumnado: Alumnado 80 y familias 52.

Familias: ISEC bajo, más del $90 \%$ son familias becadas. El 25\% procedencia magrebí (fundamentalmente Marruecos), 30\% alumnado autóctono gitano, 30\% alumnado autóctono no gitano, 5\% procedencia portuguesa y el 10\% procede de América Latina (Perú y Colombia).

Director: este es mi cuarto curso en el centro y mi cuarto curso como director. Cuando se me asignó el destino definitivo en este centro, se me propuso desde inspección la dirección del centro.

Explica brevemente cuáles son los cambios principales que, a tu juicio, ha generado la suspensión de la asistencia a clases presenciales, tanto para el profesorado como para el alumnado.

Aunque es una obviedad, el mayor cambio es la imposibilidad de desarrollar los procesos comunicativos y de relación que de manera natural se suelen dar de manera presencial. Porque, en mi opinión, hemos de tener claro que la escuela no es solo lo que sucede en el aula. De hecho, la socialización y la gestión de los conflictos son los grandes aprendizajes que se dan en el ámbito escolar y, lógicamente, en este contexto no van a ser fácil que se den. La instrucción es solo una pequeña parte, o al menos eso debiera ser, de todo lo que sucede en los centros escolares.

¿Cómo se ha llevado a cabo el proceso de adaptación a esta nueva situación, por parte de la comunidad educativa (profesorado, alumnado, familias)? ¿ Destacarías alguna cuestión significativa en este proceso?

En nuestro caso, desde el primer momento, hemos tenido claro que lo primordial era el factor humano y el cuidado; de las familias y el alumnado por parte del profesorado y del profesorado por parte del equipo directivo. Con esa premisa hemos adoptado todas las medidas necesarias para desarrollar esas ideas.

Tenemos claro que hemos de adaptarnos a cada una de las circunstancias; esta idea es uno de los signos de identidad de nuestro centro: consideramos que el sistema (la escuela) se ha de adaptar a cada uno de nuestros niños y niñas y no al revés, como suele suceder en demasiadas ocasiones. Con máximas expectativas para todo el alumnado y siendo muy flexibles y adaptativos tanto «por debajo» como «por arriba». En definitiva, decantándonos por la inclusión real y con una visión clara de equidad. 
Destacaría, quizás, la rapidez con la que nos hemos «adaptado» a la situación. En algunos centros, como el nuestro, la incertidumbre es habitual en nuestro día a día. Es por eso por lo que, en circunstancias de incertidumbre, como es el caso, actuamos con rapidez y agilidad a la hora de adaptarnos a cada situación. Eso no significa que salga todo bien a la primera; lo que digo es que, en vez de enquistarnos en el lamento y en la queja, nos ponemos manos a la obra desde el principio, tratando de poner en práctica las dos ideas clave de nuestro proyecto educativo: el primero lo mencionaba antes, el sistema se adapta a cada niño y niña, no al revés. Y cada persona tiene su propio ritmo de aprendizaje y sus circunstancias personales; y somos los y las docentes los que tenemos que propiciar la zona de desarrollo próximo de cada niño y niña (independientemente del curso académico en el que se encuentre).

\section{¿Cómo realizas en estas nuevas circunstancias las tareas de dirección del centro en el día a día?}

Como la mayoría de los centros, estamos trabajando mucho a través de videoconferencias, teléfono y WhatsApp. La parte más complicada, en mi opinión, es esa que se hace mucho en los equipos directivos y que consiste en ir «apagando pequeños fuegos» pero constantes. Habitualmente suelen ser temas que los solventas en pocos minutos; sin embargo, en esta situación y a través de los medios virtuales se pierde esa agilidad y efectividad.

Pero, en cualquier caso, hay mucha labor en la dirección que se realiza a través del teléfono y el correo electrónico, con lo cual, esa parte no ha cambiado mucho.

\section{¿Qué valoración puedes realizar sobre la situación actual, y los cambios que ha ge- nerado, desde tu experiencia en la gestión y dirección del centro?}

Por un lado, destacaría una paradoja que se ha dado en esta situación: los ecosistemas escolares permitían la conectividad, y por lo tanto la virtualidad. Sin embargo, esta situación ha retratado claramente cuál es la realidad. Una realidad, sobre la que, en general, no habíamos reflexionado. Es decir, teníamos la capacidad de trabajar telemáticamente, con la posibilidad de conectar con cualquier persona en el planeta; de lo que no nos habíamos percatado, al menos yo, es que un gran número de nuestras familias solo tenía esa posibilidad desde el propio centro.

En mi opinión, para lo que verdaderamente está sirviendo esta situación es para revisar las costuras de un sistema que evidentemente ha de ser repensado. Pero no en lo virtual, sino en su conjunto. En estos momentos, si hay aspectos que no fluyen adecuadamente, no solo es por lo que comentaba al principio de esta pregunta. La conectividad y la posibilidad de disponer de dispositivos electrónicos es evidente que habrá que asegurarla. En nuestro caso, por ejemplo, al inicio de este confinamiento, el $30 \%$ del alumnado no contaba con la mencionada conectividad a internet y un $45 \%$ no disponía dispositivos adecuados para el trabajo virtual. Este hecho se ha solventado con la colaboración del ayuntamiento de Labastida, que ha proveído de tarjetas de conexión a internet a las familias que lo necesitaban, y desde el colegio, con la colaboración del departamento de educación del Gobierno Vasco, se han prestado portátiles.

Pero lo importante, en mi opinión, es qué hacemos y qué haremos con lo que sucede dentro del mencionado ecosistema virtual; porque no todo vale. Y, según cómo abordemos el asunto, estaremos revitalizando el papel de la escuela y de los docentes; o, por el contrario, poniendo en peligro su existencia. 


\section{¿Qué dificultades destacarías a la hora de llevar a cabo tu cometido en la dirección del centro?}

Pienso que las mismas que están teniendo todas las personas que tenemos la posibilidad de teletrabajar. Por un lado, está el factor humano, que evidentemente enriquece cualquier tipo de actividad y de asunto relacionado con las personas y con la gestión de las mismas. Obviamente, no es lo mismo tratar ciertos asuntos en persona que de manera telemática o telefónica. Por ejemplo, cuando lideras reuniones del equipo docente, donde hay que tomar decisiones de calado, son muy importantes, diría que fundamentales, los matices, los gestos, las posturas, etc. que allí suceden. Estar atento a dichos elementos es una de las claves para intentar consensuar ideas, rectificar en algunas propuestas, hacer que las personas se sientan escuchadas, etc. Es evidente que a través de una videoconferencia es muy difícil ser consciente de ello.

Por otro lado, está la conciliación familiar. Si además de trabajar desde casa, tienes que estar pendiente y al cuidado de hijas pequeñas, por ejemplo, resulta muy complicado el día a día.

\section{¿Señalarías algún tipo de cambio significativo en la relación profesional con el profe- sorado de tu centro?}

Lo mencionaba en la pregunta anterior: la imposibilidad de estar cara a cara para tratar ciertos asuntos. Pienso que estamos siendo eficientes y estamos solventando la situación mucho mejor de lo que en un primer momento pudiéramos pensar, pero la parte humana es la que da sentido y verdadera calidad a una escuela y a todo lo que sucede en ella.

Por otro lado, y quizás porque siempre hemos fomentado el liderazgo distribuido, los pequeños grupos de trabajo con los que estamos funcionando en el centro están siendo muy autónomos y operativos.

Ahora te pedimos que hagas un breve balance sobre cuestiones relativas a las respuestas ante una experiencia de confinamiento y de teletrabajo y los efectos de esta situación.

Por lo que veo en general es que lo que está sucediendo en esta situación de confinamiento, haciendo referencia a lo que los centros escolares estamos ofreciendo al alumnado, no deja de ser lo que habitualmente sucede en el día a día de nuestros centros escolares. En ellos está muy extendido un planteamiento de escuela de talla única en el que es el niño o la niña quien se debe ajustar al sistema y no al revés (independientemente de las condiciones personales, las capacidades, diversas dificultades, aspectos madurativos, etc.). Y, en mi opinión, en estas circunstancias, a pesar de que lo virtual puede ser una gran oportunidad para la necesaria personalización de los aprendizajes (si de inclusión estamos hablando), seguimos en el paradigma de escuela de talla única que mencionaba.

Sí que es cierto que es imposible, e incluso contraproducente, cambiar de paradigma de manera inmediata y automática, pero al menos sí que nos debe servir para reflexionar e iniciar una transformación al respecto: la escuela de talla única no tiene sentido, ni cabida, en un planteamiento de escuela inclusiva y, por lo tanto, en mi opinión, ha de ser repensada; porque una escuela es escuela si es inclusiva y no deja a nadie atrás.

Otra de las conclusiones que podemos extraer de este confinamiento, y que pienso que para nadie ha supuesto una sorpresa, es que, como siempre, las familias vulnerables son las más perjudicadas; y obviamente, en circunstancias extremas como esta, mucho más. Y como decía anteriormente, no podemos permitir que nadie se quede atrás. Estas circunstancias están evidenciando el necesario carácter compensatorio que ha de tener la escuela. 
Asimismo, otro de los asuntos fundamentales en este periodo está siendo el de las viviendas y los recursos materiales que en ellas hay. La calidad y habitabilidad de las mismas, la conectividad, los dispositivos electrónicos con los que se cuentan, la posibilidad de alimentarse adecuadamente, etc.

¿Encuentras diferencias en la respuesta del profesorado ante la situación actual? ¿̨Responde todo el profesorado de la misma manera a la hora de realizar la actividad docente en línea? Si la respuesta es diferente, ¿̇a qué crees que se debe? ¿Qué efectos produce esta situación en el profesorado?

Es evidente, que al igual que sucede en la escuela presencial, las prácticas y los enfoques docentes suelen ser muy diferentes entre ellos. En el confinamiento no iba a ser de otra manera. Por eso son tan importantes los proyectos educativos de los centros. La educación que recibe un niño o una niña a lo largo de su vida, no puede depender de la buena o mala suerte de coincidir con uno u otro docente año tras año. El filósofo Daniel Innerarity, en el ámbito político, habla de generar sistemas inteligentes. Estoy muy de acuerdo con ese concepto y esa visión para la educación. Desde este punto de vista, cada centro escolar sería un sistema inteligente donde se generen mecanismos e inercias que aseguren algunos aspectos fundamentales. Por supuesto que cada docente tendrá su manera particular de proceder. Pero lo hará con un planteamiento alineado con el proyecto de centro. Un proyecto que habrá sido gestado por toda la comunidad escolar y que habrá sido adaptado al contexto.

Tampoco podemos obviar, por un lado, la necesidad de formación en el manejo de TIC y, por otro, el tipo de utilización de las nuevas tecnologías en las aulas. Habitualmente, la tecnología ha ido entrando en los centros de manera más o menos masiva, y posteriormente hemos comenzado a pensar sobre su uso y su encaje en el trabajo del día a día. Sin embargo, y posiblemente debido a ese orden erróneo de su implantación, no hemos sabido, en general, encontrar la armonía y la eficiencia en su uso diario. Y lógicamente este hecho se evidencia en este periodo extremo en el que nos encontramos.

\section{¿Se ha puesto en marcha algún proceso de formación del profesorado para orientar su actividad docente ante el cambio producido por el paso de la docencia presencial a la docencia en línea? Si es así, ¿̇puedes hacer alguna valoración sobre esta expe- riencia?}

En nuestro caso, por un lado, seguimos en contacto estrecho con nuestra asesora de referencia del Berritzegune, con la que, en caso, de ver necesario algún tipo de formación, ella y el equipo de asesoramiento nos lo pueden facilitar. Por otro lado, gracias a la buena relación profesional y de colaboración que mantenemos con la Facultad del Profesorado de la Universidad de Mondragón, hemos realizado algunas sesiones de formación telemática con ellos en torno a la implementación del portfolio. Es una herramienta sobre la que estamos trabajando y formándonos durante todo el curso y ahora hemos encontrado la necesidad, y la oportunidad, de profundizar en su variante digital (ePortfolio), que nos puede permitir la recogida de evidencias, desde un punto de vista de evaluación formativa, para realizar un adecuado seguimiento de la evolución de nuestro alumnado. 
¿Qué respuestas estás encontrando por parte de las familias para acompañar las tareas educativas de los hijos escolarizados en la educación obligatoria? ¿Es la misma por parte de las familias? ¿¿Encuentras diferencias entre unas y otras? Si es así, ¿a qué crees que es debido?

Las respuestas son muy variadas. Normalmente están relacionadas con el tipo de acompañamiento que habitualmente se da en el curso ordinario. Obviamente, están condicionadas por aspectos técnicos, de salud y emocionales que puedan generarse por la situación que estamos viviendo, pero, en general, las familias que podían y querían ayudar a sus hijos e hijas, ahora también lo están haciendo; sin embargo, aquellas otras con las que, anteriormente, teníamos dificultades ahora también sucede lo mismo e incluso, en algunos casos, agravado por las razones que comentaba.

¿Qué respuestas estás encontrando por parte de las y los estudiantes a la hora de realizar las tareas educativas cotidianas en una situación de educación en línea?

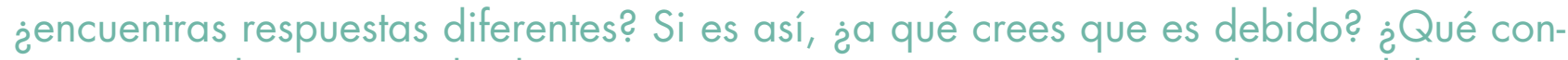
secuencias destacarías desde tu conocimiento y experiencia como director del centro? Es un hecho que aquellos grupos que habitualmente trabajan de manera colaborativa y virtual, están teniendo muchas menos dificultades. En nuestro caso el alumnado del 3er ciclo de Primaria está muy habituado a esta manera de trabajar. Para ellos y ellas no ha supuesto ningún inconveniente desde un punto de vista técnico.

Sin embargo, a medida que vas bajando de edad, el asunto se complica y, en muchos casos, entran las habilidades que tienen las madres y los padres para ayudar al alumnado desde las casas. En cualquier caso, la disposición del alumnado está siendo muy buena. Porque lo que es evidente es que la escuela es mucho más que este tipo de dinámicas muy limitadas que se pueden generar en el entorno virtual.

\section{¿Qué respuesta están recibiendo los centros por parte de la administración educativa (consejería de educación, inspección educativa...) ante esta situación?}

Creo que, para todos y todas, esta situación ha sido, y está siendo, un absoluto shock. Ante una conmoción de este tipo las primeras reacciones suelen ser imprecisas e incluso desmesuradas, fruto de la precipitación. Por eso, pienso, que en todos los casos (en el de los centros escolares y el profesorado también), sería injusto analizar las primeras decisiones que se pudieron tomar: que en general fueron desproporcionadas y exageradas, con el afán de intentar dar la mejor respuesta posible.

Dicho esto, y obviando esos primeros días, en nuestro caso la inspección educativa, que de alguna manera es el contacto directo con el departamento de educación, nos está ayudando mucho; está sirviéndonos de gran apoyo. Por un lado, obviando la burocracia excesiva, que cuando se da suele dificultar el desarrollo de los aspectos más importantes; y, por otro lado, transmitiéndonos mucha seguridad y confianza. Aunque diría que, en general, el modelo de inspección al que, al menos desde que soy director, estamos acostumbrados no difiere demasiado al que en estos momentos estamos recibiendo: control, por supuesto; pero dándonos mucha autonomía, mucha flexibilidad y mucha oportunidad de discusión crítica.

Asimismo, se nos está facilitando la posibilidad de disponer de equipos informáticos para prestar al alumnado que en casa no tiene.

¿Cuáles son las principales conclusiones que extraes de esta experiencia educativa? Creo, fundamentalmente, que estamos ante una gran oportunidad. Pienso que nos encontramos ante la 
posibilidad de repensar y rediseñar la escuela a largo plazo. Sería un error abordar el asunto centrándonos exclusivamente en el periodo de confinamiento (que va a ser un periodo muy corto de nuestras vidas). Aspectos del sistema educativo que llevan encima de la mesa mucho tiempo y que, por una u otra razón, no han sido abordados son esas costuras del sistema que en estas circunstancias están reflejando su debilidad. Por ejemplo: la evaluación (y dentro de este aspecto diferenciar la calificación de un verdadero proceso evaluador que nos sirva para acompañar al alumnado), el currículum (absolutamente inabarcable y que por su diseño impide la profundización de los aprendizajes y fomenta la superficialidad de los mismos), la función docente centrada exclusivamente en la instrucción, la distribución del alumnado teniendo en cuenta el año de nacimiento, la necesaria personalización de los aprendizajes, los tiempos en la escuela (condicionados por un planteamiento de compartimentación de los aprendizajes en asignaturas), la necesaria armonía que ha de existir entre lo virtual y lo presencial, etc.

Desde luego, no va a ser fácil, pero seriamos unos irresponsables si cuando nos recuperemos de todo esto no pensamos en profundidad en relación a lo mencionado. En que la escuela, hoy por hoy, deja a demasiadas personas atrás, que habitualmente son las más vulnerables y que en definitiva genera una reproducción del sistema social desigual en el que vivimos, y que será mucho más desigual después de este periodo de tiempo.

Nunca más que ahora ha cobrado valor la escuela. Esa escuela donde existen conflictos de convivencia, gracias a los cuales vamos convirtiéndonos en ciudadanos y ciudadanas que saben abordar ese tipo de situaciones. En estas circunstancias han desaparecido los conflictos del día a día, «del roce»; es mucho más cómodo, desde luego, pero estamos coartando a la escuela de uno de sus pilares fundamentales.

También diría, para acabar, que debemos estar alerta a los que, no tengamos ninguna duda, van a tratar de sacar provecho de esta situación y van a intentar vendernos plataformas y dispositivos que pueden generar entornos individualizadores y deshumanizados. Y que vuelva a suceder lo que comentaba anteriormente: que implantemos, primero, masivamente dispositivos, por la situación de shock en la que nos encontramos; y que posteriormente tengamos que pensar qué hacer con ellos. Tenemos que ver más allá de la emergencia y la urgencia del momento; a pesar de que, obviamente, no es nada fácil.

Señala si crees que está teniendo algún impacto en términos de igualdad o desigualdad para el alumnado y, en caso afirmativo, en qué sentido.

Obviamente. De alguna manera, a lo largo de todas mis respuestas anteriores, he mantenido esta idea como eje transversal. Si en condiciones habituales esa desigualdad es un hecho, en circunstancias extremas, y esta lo es, las personas más desfavorecidas son aún más, si cabe, las mayormente perjudicadas.

Ahora te pedimos que hagas un breve balance de esta experiencia de confinamiento y de teletrabajo educativo en relación a las siguientes cuestiones:

Principales debilidades y dificultades, situaciones de estrés, de conflicto entre el profesorado y entre el alumnado.

La mayor dificultad nos está suponiendo el contactar con algunas familias que no están por la labor. A pesar de que nuestro planteamiento es de acompañamiento, e incluso de apoyo emocional, está siendo muy complicada la comunicación con algunas de ellas. No son muchas, pero son esas que no podemos permitir que se queden atrás.

Luego están los problemas técnicos que hay que ir solventando con algunas familias, pero el profe- 
sorado está realizando un trabajo inconmensurable en ese sentido: familias que jamás han utilizado un ordenador están realizando videoconferencias comunitarias.

Pienso que en un mes estamos avanzando en competencia digital más que en los últimos lustros.

Aspectos positivos, algo que puedas extraer como una ventaja en medio de esta situación compleja, alguna reflexión en términos de experiencia profesional y crecimiento personal.

Pienso que si hacemos bien las cosas, la escuela, la de verdad (esto es un sucedáneo) va a salir reforzada.

Y, por otro lado, la profesionalidad de todo el profesorado del colegio que dirijo. Estamos demostrando, y poniéndolo en práctica todos los días, que en cualquier circunstancia no queremos que nadie se quede atrás. Y evidenciando que la labor docente tiene un componente mayor de ética que de técnica.

\section{Y en términos emocionales, afectivos y personales, ¿̇cómo lo estás viviendo? ¿̇cómo lo estás gestionando?}

Creo que, como todas las personas, tratando de llevar esto lo mejor posible. Ya que compatibilizar teletrabajo con el cuidado y atención de hijas pequeñas no es nada fácil.

Y también, lo digo sinceramente, valorando el estatus privilegiado en el que en mi caso, al menos, me encuentro: toda la gente cercana está bien de salud, vivimos en una buena casa, no tenemos problemas económicos y de trabajo, etc.

\section{Qué aprendizaje extraes de esta experiencia de cara al futuro.}

Pienso que, en estas circunstancias, más que nunca se ha evidenciado, por un lado, la necesidad de tener unos servicios públicos de calidad, con un sistema de protección social adecuado y por otro lado, la importancia de lo comunitario, de lo colaborativo.

Todo esto debiera ser abordado en la escuela, de manera curricular: promoviendo ciudadanos y ciudadanas comprometidos que sean conscientes de que el utópico, en estos momentos, bienestar social generalizado depende de todos nosotros que, con nuestras decisiones personales en el día a día, lo fomentamos o, por el contrario, hacemos que no sea posible.

Te pedimos también que valores las consecuencias y el impacto educativo y social de esta crisis a corto y medio plazo, una vez que se supere la situación.

En general, no creo que en tres meses sin clase vaya a cambiar nada sustancialmente. Es decir, cuando volvamos, todo se irá ajustando de manera paulatina y los niños y niñas irán adecuándose al nuevo ritmo de la escuela presencial. Volverán a surgir los, necesarios, conflictos del día a día y en mi opinión, todo volverá a la situación habitual.

Ahora bien, ahí está la clave de todo esto: podemos optar por abordar la vuelta con mucha calma y con una visión de cuidado emocional del alumnado o, por el contrario, nos pueden entrar las prisas y considerar que hay que «recuperar» en 3 semanas (o en el tiempo que se considere) «todo lo que no se ha hecho» en el periodo de confinamiento.

Asimismo, aquellos centros y docentes que ya estaban realizando transformaciones y prácticas verdaderamente inclusivas (o al menos iban en esa dirección) este periodo les servirá como acicate y como filtro que constate que ciertos cambios son necesarios y urgentes. 
Mi mente pesimista piensa que en general se volverá al punto de partida en el que estábamos y que olvidaremos rápidamente todas las necesidades y urgencias que en estos momentos estamos viendo claramente; pero (mente optimista), si aquellos docentes y centros educativos que llevamos tiempo tratando de implementar actuaciones disruptivas en aras de la equidad y la inclusión, somos apoyados por la administración y la inspección, e investigados por universidades, para poder mejorar nuestros proyectos educativos y para que posteriormente se divulguen en toda la comunidad educativa, será un verdadero logro y nos permitirá seguir caminando hacia la escuela ideal que nuestros niños y niñas se merecen. 


\section{Jefe de Estudios del IES Práxedes Mateo Sagasta, Logroño, 55 años}

Describe brevemente las características del centro educativo (nombre, ubicación, niveles educativos, número de profesorado y de alumnado, perfil aproximado del profesorado -sexo, edad- y del estudiantado -variables socioeconómicas, población inmigrante, etc.-. Indica cuánto tiempo llevas trabajando en el centro educativo y desde cuándo eres director del mismo.

Nombre del centro: IES Práxedes Mateo Sagasta

Ubicación: calle Luis de Ulloa, 37, Logroño, La Rioja

Enseñanzas y alumnado: es un centro especial porque además de la enseñanza de ESO y Bachillerato tenemos, en horario vespertino y nocturno, Bachillerato de adultos. Por la mañana hay unos 630 alumnos y por la tarde unos 200. A nuestro centro acuden alumnos y alumnas de todos los barrios de Logroño, lo que hace que haya una gran diversidad, tanto a nivel socioeconómico como de origen. Hay alumnos de más de 30 nacionalidades, o de origen, distintas.

Jefa de Estudios: llevo 9 años trabajando en el Sagasta y 32 como docente.

Explica brevemente cuáles son los cambios principales que, a tu juicio, ha generado la suspensión de la asistencia a clases presenciales, tanto para el profesorado como para el alumnado.

¿Qué ha cambiado? Todo. La educación, tal como la entendíamos, ya no es posible porque se basaba en el contacto directo con el alumnado, en ver sus caras sus reacciones. Ahora nos comunicamos, pero no hay una comunicación que permita conocer qué está pensando o qué reacción has provocado en los alumnos.

¿Cómo se ha llevado a cabo el proceso de adaptación a esta nueva situación, por parte de la comunidad educativa (profesorado, alumnado, familias)? ¿ Destacarías alguna cuestión significativa en este proceso?

El proceso de adaptación a esta nueva situación se ha hecho muy rápidamente, si nos referimos a las cuestiones meramente técnicas. Ha sido increíble como, tanto profesorado como alumnado en su mayoría, nos hemos hecho usuarios de las nuevas tecnologías. Por supuesto que todos usábamos ya estas nuevas tecnologías pero, por lo menos en mi caso y creo que esto es en una mayoría, el uso era para hacer apuntes, un examen, una presentación y ya. Ahora nos conectamos, resolvemos problemas de tipo administrativo, que a mí como Jefa de Estudios me llegan, con las nuevas tecnologías. Yo nunca hubiera creído esta capacidad de aprender y adaptarnos al uso de estas herramientas.

¿Cómo realizas en estas nuevas circunstancias las tareas de dirección del centro en el día a día?

Realizamos video conferencias, tenemos creados distintos grupos de trabajo en plataformas, mantenemos las reuniones semanales que ya teníamos, con tutores, CCP, etc. Nos ponemos en contacto también por teléfono, ahora el uso de la «nube» para compartir documentos es muy importante. 
¿Qué valoración puedes realizar sobre la situación actual, y los cambios que ha generado, desde tu experiencia en la gestión y dirección del centro?

Todos estamos dedicando muchas horas, tanto a la gestión del centro por parte de los equipos directivos como los profesores para continuar con las clases, pero la sensación es de no poder abarcar todos los problemas.

En la gestión del centro ahora los problemas son diferentes de los habituales. Sobre todo tenemos que resolver problemas de alumnos que no pueden seguir la enseñanza on-line por diversos motivos. Primero hemos tenido que detectar qué alumnos tenían problemas, pero no nos ha sido posible resolver todos los casos.

Ahora el problema que tenemos es la inseguridad que genera en el profesorado y en el alumnado cómo va a ser el fin de curso. La Administración no ha dado todavía unas directrices claras y esto crea mucha inseguridad e incertidumbre.

\section{¿Qué dificultades destacarías a la hora de llevar a cabo tu cometido en la dirección del centro?}

La falta de instrucciones claras por parte de la Administración educativa.

¿Señalarías algún tipo de cambio significativo en la relación profesional con el profesorado de tu centro?

Ahora no tengo un contacto habitual con todos. Tengo contacto semanal con los tutores y con los profesores que tienen algún problema, pero ya no hay el contacto diario o casi diario con todos los profesores. Lo mismo ocurre con los alumnos, solo tengo contacto con mis alumnos que son solo los de un grupo.

Ahora te pedimos que hagas un breve balance sobre cuestiones relativas a las respuestas ante una experiencia de confinamiento y de teletrabajo y los efectos de esta situación.

La respuesta de la ciudadanía, y del profesorado y alumnado en particular, está siendo ejemplar. Es algo que me sorprende y me hace tener esperanzas en la vida más allá del confinamiento.

El teletrabajo ha entrado en nuestras vidas y todos nos hemos adaptado. Alumnos y profesores cumplen con el horario de clases que tenían y se hacen tareas. Pero todos tenemos la sensación de que este esfuerzo tiene ahora unos resultados muy diferentes de los que se esperan de la escuela. Nuestra labor principal con los estudiantes no es tanto académica como de acompañamiento en esta difícil situación. Creo que los alumnos se sienten más acompañados y con la enseñanza on-line una parte de su vida, la de ser estudiantes, continua o da apariencia de continuar.

¿Encuentras diferencias en la respuesta del profesorado ante la situación actual? ¿ Responde todo el profesorado de la misma manera a la hora de realizar la actividad docente en línea? Si la respuesta es diferente, ¿̇a qué crees que se debe? ¿̨ué efectos produce esta situación en el profesorado?

El profesorado, en general, ha respondido desde el primer día. Cada uno utiliza diversas herramientas de todas las que disponemos. Hay profesores que son más avezados y hacen vídeos, y otros solo mandan apuntes y tareas. Pero todos han contactado con sus alumnos.

La diferencia está en la competencia digital, que todos hemos mejorado, pero la situación de partida no era homogénea. 
¿Se ha puesto en marcha algún proceso de formación del profesorado para orientar su actividad docente ante el cambio producido por el paso de la docencia presencial a la docencia en línea? Si es así, ¿̇puedes hacer alguna valoración sobre esta experiencia?

Sé que se han convocado cursos on-line, pero yo no he participado en ninguno.

¿Qué respuestas estás encontrando por parte de las familias para acompañar las tareas educativas de los hijos escolarizados en la educación obligatoria? ¿̇Es la misma por parte de las familias? ¿Encuentras diferencias entre unas y otras? Si es así, ¿a qué crees que es debido?

Las familias responden de muy diversa manera. En general ayudan y apoyan a sus hijos e hijas, pero hay una minoría de familias que no pueden apoyar a sus hijos por su desconocimiento y por otros problemas, económicos y sociales. El principal problema es que esto es en los cursos inferiores, $1^{\circ}$ y $2^{\circ}$ de ESO, donde se dan más casos de familias que no pueden ayudar a sus hijos, y son estos alumnos los que tienen una menor competencia digital.

¿Qué respuestas estás encontrando por parte de las y los estudiantes a la hora de realizar las tareas educativas cotidianas en una situación de educación en línea? ¿̇encuentras respuestas diferentes? Si es así, ¿̇a qué crees que es debido? ¿̨ué consecuencias destacarías desde tu conocimiento y experiencia?

La respuesta no ha sido muy diferente de lo que ocurría antes: hay alumnos que hacen las tareas puntualmente y otros que no. Yo tengo muy pocos alumnos y mi caso no es significativo, pero por comentarios de profesores se que algunos alumnos que antes no hacían nada o poco ahora están respondiendo más. También hay unos pocos alumnos con los que no hemos podido contactar o el contacto ha sido mínimo. En la mayoría de los casos son alumnos que ya eran absentistas pero hay otros que es por no disponer de ordenador o de conexión a Internet, u otros problemas familiares. Nos tememos que con el paso de los días la situación económica de muchas familias empeore y esto se note en el rendimiento de los alumnos.

\section{¿Qué respuesta están recibiendo los centros por parte de la administración educativa (consejería de educación, inspección educativa...) ante esta situación?}

Bastante incoherente y poco útil. Parece que hay miedo de tomar decisiones y esta situación crea mucha incertidumbre e inseguridad. A fecha de hoy, 22 de abril, todavía no sabemos si va a haber cambios en el calendario escolar.

¿Cuáles son las principales conclusiones que extraes de esta experiencia educativa? Que ojalá no se repita. No creo que sea una forma adecuada como experiencia educativa para alumnos de infantil, primaria, secundaria y bachillerato. Temporalmente se puede hacer y lo estamos haciendo, pero la educación de nuestros niños y jóvenes no puede hacerse a través de una pantalla de forma habitual y continuada.

Señala si crees que está teniendo algún impacto en términos de igualdad o desigualdad para el alumnado y, en caso afirmativo, en qué sentido.

Sí está teniendo impacto en términos de igualdad. La Escuela Pública, a la que me honra pertenecer, tiene 
como uno de sus principios básicos la equidad, es un motor para disminuir las diferencias que por origen o nacimiento hay entre nuestros alumnos. Esto se ha perdido en buena parte. Ahora es muy importante la labor de las familias, las herramientas de las nuevas tecnologías que dispongan los alumnos en casa, la conexión a Internet, el tener una mesa de trabajo aislada del resto de tu familia, y para eso necesitas vivir en una casa no muy pequeña, etc.

A las carencias en estos aspectos de muchos de nuestros alumnos no podemos darles solución. Solo podemos dar ordenadores y tarjetas con datos, pero ¿cómo y dónde vas a usar una tablet en una casa de $50 \mathrm{~m}^{2}$ donde viven 5 ó 6 personas? No solo es la brecha digital, es la brecha socioeconómica la que ahora se manifiesta claramente y perjudica más que nunca.

Ahora te pedimos que hagas un breve balance de esta experiencia de confinamiento y de teletrabajo educativo en relación a las siguientes cuestiones:

Principales debilidades y dificultades, situaciones de estrés, de conflicto entre el profesorado y entre el alumnado.

El estrés se produce por el uso o el no uso de las herramientas online. Hay profesores que se quejan de que los alumnos unos días se conectan y otros días no. También ha surgido algún conflicto por no poder dar una respuesta clara a cómo va a ser la evaluación.

Aspectos positivos, algo que puedas extraer como una ventaja en medio de esta situación compleja, alguna reflexión en términos de experiencia profesional y crecimiento personal.

La gran capacidad de adaptación y cómo todos nos hemos esforzado para continuar dando clases, los profesores, y siguiendo las clases, los alumnos. Es un ejemplo claro de la capacidad de superación ante situaciones inesperadas y difíciles.

\section{Y en términos emocionales, afectivos y personales, ¿̇cómo lo estás viviendo? ¿̇cómo lo} estás gestionando?

Pues a ratos. Me produce ansiedad no saber cuándo va a acabar el confinamiento y qué nos espera el futuro. El estar en casa no me supone un gran esfuerzo, soy una persona muy casera. Pero ¿qué va a ser de nuestros alumnos? ¿Cómo les va a influir esta situación?

\section{Qué aprendizaje extraes de esta experiencia de cara al futuro.}

Tenemos que valorar más lo comunitario, ser menos individualistas, defender y proteger todos los servicios públicos y convertir en servicio público algunos sectores en manos de la empresa privada actualmente.

Te pedimos también que valores las consecuencias y el impacto educativo y social de esta crisis a corto y medio plazo, una vez que se supere la situación.

Esta valoración es la que no soy capaz de hacer y por ello me genera ansiedad. Me considero una optimista realista pero ahora no veo más allá de que mañana continúa el confinamiento. 
Finalmente, nos gustaría preguntarte por alguna otra cuestión, comentario, sugerencia, que quieras hacer para terminar.

Espero que cuando esto acabe no se nos olvide en un par de meses y cambiemos de verdad nuestra forma de vivir. Las imágenes de delfines en las playas, incluso en los canales de Venecia, nos deben servir para concienciarnos de que la naturaleza se regenera cuando los humanos nos refugiamos en nuestras madrigueras y que no tenemos el derecho de destruirla. 